\title{
CEA clearance pattern as a predictor of tumor response to neoadjuvant treatment in rectal cancer: a post-hoc analysis of FOWARC trial
}

Huabin Hu ${ }^{1 \dagger}$, Jin Huang ${ }^{2+}$, Ping Lan ${ }^{3,4}$, Lei Wang ${ }^{3,4}$, Meijin Huang ${ }^{3,4}$, Jianping Wang ${ }^{3,4^{*}}$ and Yanhong Deng ${ }^{1^{*}}$

\begin{abstract}
Background: The clinical factors that accurately predict the response to preoperative treatment in rectal cancer were yet unknown. The carcinoembryonic antigen (CEA) clearance pattern during neoadjuvant treatment has been developed and the predictive value explored in rectal cancer patients with elevated CEA levels (>5 ng/mL).

Methods: The training cohort was derived from the FOWARC prospective phase III trial, and 71/483 eligible patients were included. The validation cohort consisted of 75/587 consecutive rectal cancer patients from Xiangya Hospital between 2014 and 2015. The kinetic changes in serum CEA were measured at different time points during the neoadjuvant treatment. An exponential trend line was drawn using the CEA values. The patients were categorized into two groups based on the $R^{2}$ value of the trend line, which indicates the correlation coefficient between the exponential graph and measured CEA values: exponential decrease group $\left(0.9<R^{2} \leq 1.0\right)$ and non-exponential decrease group $\left(R^{2} \leq 0.9\right)$.

Results: In multivariate analysis, the patients in the CEA exponential decrease group had significantly high adequate rate of downstaging (ypT0-2NOMO), and pathologic complete response (pCR) rates after neoadjuvant treatment in the training cohort. The predictive values of the CEA clearance pattern for tumor downstaging and $\mathrm{PCR}$ were further confirmed in an independent validation cohort.

Conclusions: The CEA clearance pattern was an independent predictor of tumor response to neoadjuvant treatment in patients with rectal cancer. It might serve as an adjunct in the assessment of complete clinical response and guide individualized treatment strategies.
\end{abstract}

Trial registration: NCT01211210.

Keywords: Rectal cancer, Carcinoembryonic antigen, Neoadjuvant treatment, Pathologic complete response

\section{Background}

Neoadjuvant chemoradiation therapy followed by total mesorectal excision (TME) for locally advanced rectal cancer has been considered as the optimal management strategy owing to increased control of local disease,

\footnotetext{
* Correspondence: wangjpgz@126.com; rebosome@qq.com ${ }^{\dagger}$ Huabin $\mathrm{Hu}$ and Jin Huang contributed equally to this work.

${ }^{3}$ Department of Colorectal Surgery, The Sixth Affiliated Hospital of Sun Yat-Sen University, Yuancunheng 2nd Road, Guangzhou 510655, People's Republic of China

${ }^{1}$ Department of Medical Oncology, The Sixth Affiliated Hospital of Sun Yat-Sen University, Yuancunheng 2nd Road, Guangzhou 510655, People's Republic of China

Full list of author information is available at the end of the article
}

decreased toxicity, and greater sphincter preservation rates [1-4]. Nevertheless, great differences in treatment response are continually observed among treated patients. Tumor downstaging can be obtained only in half of the cases, and a pathologic complete response (pCR) is $10-30 \%[5,6]$.

An optimal knowledge of the factors that predict response to preoperative treatment may eventually lead to the development of individualized, risk-adapted treatment strategies for rectal cancer patients. Biochemical markers and clinical characteristics including serum

(c) The Author(s). 2018 Open Access This article is distributed under the terms of the Creative Commons Attribution 4.0 International License (http://creativecommons.org/licenses/by/4.0/), which permits unrestricted use, distribution, and reproduction in any medium, provided you give appropriate credit to the original author(s) and the source, provide a link to the Creative Commons license, and indicate if changes were made. The Creative Commons Public Domain Dedication waiver (http://creativecommons.org/publicdomain/zero/1.0/) applies to the data made available in this article, unless otherwise stated. 
carcinoembryonic antigen (CEA), tumor length, and circumferential tumor extent have been investigated as predictors [7-12].

CEA is a cell surface-anchored glycoprotein that was first identified in human colon cancer tissue extracts [13]. The levels CEA in pre- and post-treatment were described as independent predictive factors of response to neoadjuvant treatment $[9,14]$. Other studies showed that the normalization of CEA levels after neoadjuvant treatment was correlated with PCR $[15,16]$. The data on the evaluation of the dynamic changes in CEA levels suggested that a predictive indicator during neoadjuvant treatment of rectal cancer was scarce. Thus, the present study aimed to investigate the pattern of serum CEA clearance as a predictive tool for tumor response in rectal cancer patients receiving neoadjuvant treatment.

\section{Methods}

\section{Patient population}

The training cohort was derived from the FOWARC randomized prospective phase III trial exploring mFOLFOX6- and fluorouracil-based preoperative chemoradiotherapy in the locally advanced rectal cancer [17]. The patients were eligible for the present investigation if they fulfilled the following criteria: 1) received neoadjuvant chemoradiation followed by TME resection, 2) baseline CEA level was elevated ( $\geq 5 \mathrm{ng} / \mathrm{mL})$, and 3) follow-up CEA values (2nd, 4th, 6th, 8th and 14th week from the beginning of the preoperative treatment) were available during the study. Finally, 71 patients were included in the training cohort (Additional file 1: Figure S1). The validation cohort consisted of 75/587 consecutive rectal cancer patients undergoing fluorouracil-based neoadjuvant chemoradiation and TME resection between 2014 and 2015 at the Xiangya Hospital of Central South University. The inclusion and exclusion criteria were similar to that of the FOWARC trial and those previously described in the training cohort, except that CEA was measured at four time points, including the baseline, 2nd, 6th, and 12th weeks from the start of the preoperative treatment (Additional file 2: Figure S2).

\section{Treatment}

In the training cohort, patients in the fluorouracilradiotherapy group received preoperative treatment with five cycles of infusional fluorouracil [leucovorin $400 \mathrm{mg} / \mathrm{m}^{2}$ intravenously, followed by fluorouracil $400 \mathrm{mg} / \mathrm{m}^{2}$, and fluorouracil $2.4 \mathrm{~g} / \mathrm{m}^{2}$ by $48 \mathrm{~h}$ continuous intravenous infusion (de Gramont regimen)] with concurrent radiotherapy during cycles 2-4. The mFOLFOX6radiotherapy group was administered similar treatment as the fluorouracil-radiotherapy group plus oxaliplatin $85 \mathrm{mg} / \mathrm{m}^{2}$ intravenously on day 1 of each chemotherapy cycle. Radiotherapy was administered at 1.8-2.0 Gy for
23-28 fractions over 5-6 weeks and a total dose of 46.0-50.4 Gy [17]. In the validation cohort, patients underwent 5 weeks of radiotherapy treatment at $50 \mathrm{~Gy} / 25$ fractions with concurrent capecitabine $800 \mathrm{mg} / \mathrm{m}^{2}$ two times daily for 5 consecutive days/week.

\section{Pathological assessment}

All resection specimens were examined according to a standardized protocol that included TNM classification according to the American Joint Committee on the Cancer-International Union Against Cancer (seventh edition). pCR was defined as the absence of viable cells in the primary tumor and lymph nodes (ypT0N0) as evaluated by two pathologists blinded to the treatment group. Tumor regression grade (TRG) was performed semi-quantitatively by determining the amount of viable tumor versus fibrotic tissue that ranged from the lack of tumor regression to complete response with no viable tumor identified. The four groups of TRG classification were as follows: grade 0 , total regression (no viable tumor cells; fibrotic mass only); grade 1 , good regression (dominant fibrosis outgrowing tumor mass [ie, more than $50 \%$ tumor regression]); grade 2, moderate regression (dominant tumor mass with obvious fibrosis in 26 to $50 \%$ of tumor mass); grade 3 , no regression or minor regression (dominant tumor mass with obvious fibrosis in $25 \%$ or less of the tumor mass) [18]. In the present study, good regression was defined as TRG $0-1$, and good tumor downstaging was defined as ypT0-2N0M0 (ypStage0-1).

\section{CEA analysis and clearance}

All of the serum CEA assays were performed within one laboratory by the Elecsys 2010 electrochemiluminescence immunoassay (Roche Diagnostics) in which the reference normal range was $0-5 \mathrm{ng} / \mathrm{ml}$. The serum concentration of CEA was measured at the baseline (within 7 days before preoperative treatment start) and then during the preoperative treatment (training cohort: at 2nd, 4th, 6th, 8th, and 14th weeks from the start of preoperative treatment; validation cohort: at the 2nd, 6th, and 12th weeks from the start of preoperative treatment).

Our initial hypothesis postulated that the CEA decay and production, rendered an exponential distribution depending on the state of tumor activity. If the tumor is more sensitive to the neoadjuvant chemoradiation, the CEA produced by the rectal cancer tissue is suppressed more thoroughly. As a result, the clearance pattern of CEA follows the classical the exponential kinetics format [19-22].

The exponential curves were drawn based on the trend line, and $R^{2}$ values calculated. $R^{2}$ indicates the correlation coefficient between the trend line illustrating the exponential decrease and the measured CEA values. 
If $\mathrm{R}^{2}=1$, all the data points will fall on the exponential line. Therefore, the closer the $\mathrm{R}^{2}$ value to 1 , more CEA values fit the exponential curve, and the patients with exponential CEA clearance potentially displayed an adequate tumor response (Fig. 1). We categorized the patients into two groups based on the $R^{2}$ values: exponential decrease group $\left(0.9<R^{2} \leq 1.0\right)$, and non-exponential decrease group $\left(R^{2} \leq 0.9\right)$.

\section{Statistical analysis}

Comparison of variables was performed with the X2 test or Fisher exact test for qualitative variables, and the Student $t$ test was used for quantitative variables. Univariate and multivariate analyses were performed by using the Student $\mathrm{t}$ test and logistic regression to identify predictors for the end points TRG, tumor downstaging and pCR. Only factors for which $p \leq 0.05$, as determined in the univariate analysis in the training cohort, were entered into the multivariate analysis in the training and validation cohort. The area under the curve (AUC) is used as a summary measure of the receiver operating characteristics (ROC) curve (which plots sensitivity versus 1-specificity) and represents the discrimination ability. AUC is expressed on a scale of 0.5 to 1 . The larger the AUC value, the better the classification effect. In this study, AUC represents the prediction accuracy of CEA clearance pattern and clinical parameters for TRG, tumor downstaging, and pCR. To determine whether the CEA was in the normal range, $5 \mathrm{ng} / \mathrm{mL}$ was used as the cutoff. For all analyses, $\mathrm{P}<<0.05$ was considered statistically significant. Statistical analyses were performed using software SPSS versions 22 (SPSS Inc. Chicago, IL, USA) and MedCalc version 13.0 (MedCalcSoftware).

\section{Results \\ Patient characteristics}

The clinicopathological characteristics of patients are shown in Table 1 . In the training cohort, 32 patients (45\%) comprised the fluorouracil-radiotherapy group, and $39(55 \%)$ formed the mFOLFOX6-radiotherapy group. The mean pre- or post-chemoradiation CEA level was 27 and $5 \mathrm{ng} / \mathrm{mL}$, respectively. The CEA level in 47 patients $(66 \%)$ dropped to the normal range $(\leq 5 \mathrm{ng} / \mathrm{mL})$ after 6-8 weeks from the completion of chemoradiation.

In the validation cohort, all patients underwent a neoadjuvant radiotherapy with concurrent capecitabine. The mean pre-chemoradiation CEA level was $14 \mathrm{ng} / \mathrm{mL}$, which was significantly lower as compared to the training cohort. The mean post-chemoradiation CEA level was $6 \mathrm{ng} / \mathrm{mL}$, and the normalization of CEA rate was $61 \%$ (46 patients).

$44 \%(31 / 71)$ and $48 \%(36 / 75)$ patients were categorized into the CEA exponential decrease group in the training and the validation cohorts, respectively. The clinicopathological characteristics were similar between the two cohorts, except gender, tumor differentiation, and

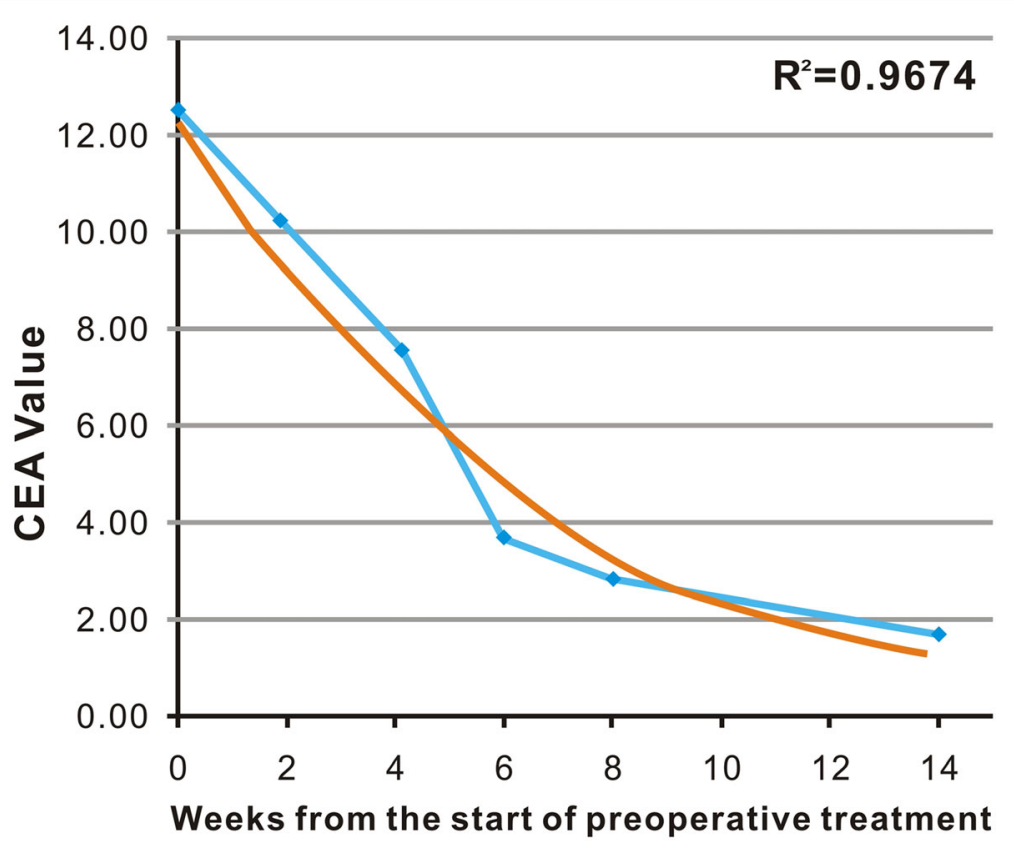

Fig. 1 An exponential trend line (blue line) was drawn using each CEA value (baseline, 2nd, 4th, 6th, 8th, and 14th week from the start of preoperative treatment). The function of the exponential curve (yellow line) was drawn. $R^{2}$ values were calculated as the deviation between the calculated curves and the measured CEA value 
Table 1 Clinicopathological characteristics of the training $(n=71)$ and validation cohorts $(n=75)$

\begin{tabular}{|c|c|c|c|}
\hline Clinicopathologic indexes & Training cohort $(n=71)$ & Validation cohort $(n=75)$ & $P$ \\
\hline Age (mean, y $\pm S D$ ) & $54 \pm 12$ & $54 \pm 12$ & 0.801 \\
\hline Gender & & & 0.011 \\
\hline Male & $47(66 \%)$ & $34(45 \%)$ & \\
\hline Female & $24(34 \%)$ & $41(55 \%)$ & \\
\hline Tumor differentiation & & & 0.005 \\
\hline Well/Moderately & $34(48 \%)$ & $56(75 \%)$ & \\
\hline Poorly/Undifferentiated & $37(52 \%)$ & $19(25 \%)$ & \\
\hline Distance from anal verge & & & 0.259 \\
\hline$\leqq 5 \mathrm{~cm}$ & $35(49 \%)$ & $30(40 \%)$ & \\
\hline$>5 \mathrm{~cm}$ & $36(51 \%)$ & $45(60 \%)$ & \\
\hline Tumor length (median; range) & $4(2-12)$ & $4(2-11)$ & 0.948 \\
\hline Circumferential extent & & & 0.113 \\
\hline$\leqq 50 \%$ & $17(24 \%)$ & $27(36 \%)$ & \\
\hline$>50 \%$ & $54(76 \%)$ & $48(64 \%)$ & \\
\hline Pretreatment T stage & & & $<0.001$ \\
\hline $\mathrm{T}_{2}$ & 0 & $6(8 \%)$ & \\
\hline$T_{3}$ & $52(73 \%)$ & $62(83 \%)$ & \\
\hline $\mathrm{T}_{4}$ & $19(27 \%)$ & 7 (9\%) & \\
\hline Pretreatment $\mathrm{N}$ stage & & & 0.119 \\
\hline $\mathrm{N}_{0}$ & $12(17 \%)$ & $21(28 \%)$ & \\
\hline $\mathrm{N}_{1}$ & $30(42 \%)$ & $34(45 \%)$ & \\
\hline $\mathrm{N}_{2}$ & $29(41 \%)$ & $20(27 \%)$ & \\
\hline Pre- treatment CEA level (mean, ng/mL; rang) & $27(5-200)$ & $14(5-57)$ & 0.001 \\
\hline Post- treatment CEA level (mean, $\mathrm{ng} / \mathrm{mL}$; rang) & $5(0.5-45)$ & $6(0.5-34)$ & 0.605 \\
\hline Normalization of post- treatment CEA Level & & & 0.541 \\
\hline Normal (<5 ng/mL) & $47(66 \%)$ & $46(61 \%)$ & \\
\hline Elevated ( $\geqq 5 \mathrm{ng} / \mathrm{mL})$ & $24(34 \%)$ & $29(39 \%)$ & \\
\hline CEA clearance pattern & & & 0.599 \\
\hline Exponential decrease $\left(R^{2} \geqq 0.9\right)$ & $31(44 \%)$ & $36(48 \%)$ & \\
\hline Non-exponential decrease $\left(R^{2}<0.9\right)$ & $40(56 \%)$ & $39(52 \%)$ & \\
\hline
\end{tabular}

The $p$ value in boldface means statistically significant, that is, less than 0.05

pre-treatment $\mathrm{T}$ stage. The validation cohort included fewer males (45 vs. 66\%), and patients exhibited poor tumor differentiation ( 25 vs. $52 \%$ ), and T4 (9 vs. $27 \%$ ) than those in the validation cohort (Table 1).

\section{Association of CEA clearance pattern with clinicopathological characteristics}

In the training cohort, we found that the patients in the CEA exponential decrease group were associated with circumferential extent $\leq 50 \%(P=0.001)$; whereas, the relationship between the CEA clearance pattern and clinicopathological parameters for the validation cohort was not statistically significant (Additional file 3: Table S1).

\section{Predictive significance of CEA clearance pattern in the} training cohort

In the training cohort, 33 patients (46\%) showed TRG 0-1, 20 patients (28\%) exhibited good downstaging, and 11 patients $(15 \%)$ had pCR. The results from our univariate analysis indicated that the tumor length was $\leq 4 \mathrm{~cm} \quad(P=0.015)$, circumferential extent $\leq 50 \%$ $(P=0.022)$, normalization of post-treatment CEA level $<5 \mathrm{ng} / \mathrm{mL} \quad(P=0.037)$, and CEA exponential decrease $(P=0.028)$ were associated with good TRG (0-1) (Table 2). The pre-treatment $\mathrm{T}$ stage (T3 vs. T4) $(P=0.009)$, well/moderate tumor differentiation $(P=0.02)$, and CEA exponential decrease $(P=0.001)$ 
Table 2 Univariate analysis of predictors for TRG in the training and validation cohorts

\begin{tabular}{|c|c|c|c|c|c|c|}
\hline \multirow[t]{2}{*}{ Variable } & \multicolumn{3}{|c|}{ Training cohort } & \multicolumn{3}{|c|}{ Validation cohort } \\
\hline & TRG 0-1 & TRG 2-3 & $P$ & TRG 0-1 & TRG 2-3 & $P$ \\
\hline No. of patients & 33 & 38 & & 33 & 42 & \\
\hline Age (mean, $y \pm S D$ ) & $52 \pm 12$ & $56 \pm 12$ & 0.199 & $55 \pm 13$ & $55 \pm 11$ & 0.827 \\
\hline Gender & & & 0.671 & & & 0.627 \\
\hline Male & $21(64 \%)$ & $26(68 \%)$ & & $16(49 \%)$ & $18(43 \%)$ & \\
\hline Female & $12(36 \%)$ & $12(32 \%)$ & & $17(51 \%)$ & $24(57 \%)$ & \\
\hline Pretreatment T stage & & & 0.655 & & & 0.854 \\
\hline $\mathrm{T}_{2}$ & 0 & 0 & & $2(6 \%)$ & $4(9 \%)$ & \\
\hline $\mathrm{T}_{3}$ & $25(76 \%)$ & 27 (71\%) & & $28(85 \%)$ & $34(82 \%)$ & \\
\hline $\mathrm{T}_{4}$ & $8(24 \%)$ & $11(29 \%$ & & $3(9 \%)$ & $4(9 \%)$ & \\
\hline Pretreatment $\mathrm{N}$ stage & & & 0.928 & & & 0.638 \\
\hline $\mathrm{N}_{0}$ & $5(16 \%)$ & $7(18 \%)$ & & $10(30 \%)$ & $11(26 \%)$ & \\
\hline$N_{1}$ & $14(42 \%)$ & $16(42 \%)$ & & $16(49 \%)$ & $18(43 \%)$ & \\
\hline $\mathrm{N}_{2}$ & $14(42 \%)$ & $15(40 \%)$ & & $7(21 \%)$ & $13(31 \%)$ & \\
\hline Tumor differentiation & & & 0.128 & & & 0.732 \\
\hline Well/Moderately & $19(58 \%)$ & $15(40 \%)$ & & $24(73 \%)$ & $32(76 \%)$ & \\
\hline Poorly/Undifferentiated & $14(42 \%)$ & $23(60 \%)$ & & $9(27 \%)$ & $10(24 \%)$ & \\
\hline Distance from the anal verge & & & 0.120 & & & 0.924 \\
\hline$\leqq 5 \mathrm{~cm}$ & $13(39 \%)$ & $22(58 \%)$ & & $13(39 \%)$ & $17(41 \%)$ & \\
\hline$>5 \mathrm{~cm}$ & $20(61 \%)$ & $16(42 \%)$ & & $20(61 \%)$ & $25(59 \%)$ & \\
\hline Tumor length & & & 0.015 & & & 0.011 \\
\hline$\leqq 4 \mathrm{~cm}$ & $19(42 \%)$ & $11(71 \%)$ & & 19 (58\%) & $12(29 \%)$ & \\
\hline$>4 \mathrm{~cm}$ & $14(58 \%)$ & $27(29 \%)$ & & $14(42 \%)$ & $30(64 \%)$ & \\
\hline Circumferential extent & & & 0.022 & & & 0.163 \\
\hline$\leqq 50 \%$ & $12(36 \%)$ & $5(13 \%)$ & & $9(27 \%)$ & $18(43 \%)$ & \\
\hline$>50 \%$ & $21(64 \%)$ & $33(87 \%)$ & & $24(73 \%)$ & $24(57 \%)$ & \\
\hline $\begin{array}{l}\text { Pre-treatment CEA level } \\
\text { (mean, ng/ml; 95\%Cl) }\end{array}$ & $29(16-43)$ & $25(17-34)$ & 0.615 & $15(11-18)$ & $14(11-16)$ & 0.548 \\
\hline Post- treatment CEA level (mean, ng/mL; 95\%Cl) & $4(1-7)$ & $6(4-9)$ & 0.233 & $5(4-7)$ & $7(4-9)$ & 0.343 \\
\hline Normalization of post- treatment CEA Level & & & 0.037 & & & 0.187 \\
\hline Normal (<5 ng/mL) & $26(79 \%)$ & $21(55 \%)$ & & $23(70 \%)$ & $23(55 \%)$ & \\
\hline Elevated $(\geqq 5 \mathrm{ng} / \mathrm{mL})$ & $7(21 \%)$ & $17(45 \%)$ & & $10(30 \%)$ & $19(45 \%)$ & \\
\hline CEA clearance pattern & & & 0.028 & & & 0.016 \\
\hline Exponential decrease $\left(R^{2} \geqq 0.9\right)$ & $19(58 \%)$ & $12(32 \%)$ & & $21(64 \%)$ & $15(36 \%)$ & \\
\hline Non-exponential decrease $\left(R^{2}<0.9\right)$ & $14(42 \%)$ & $26(68 \%)$ & & $12(36 \%)$ & $27(64 \%)$ & \\
\hline Concurrent chemotherapy & & & 0.135 & & & NA \\
\hline Capecitabine & 0 & 0 & & $33(44 \%)$ & $42(56 \%)$ & \\
\hline Infusional fluorouracil & $15(45 \%)$ & $24(63 \%)$ & & 0 & 0 & \\
\hline mFOLFOX6 & $48(55 \%)$ & $14(37 \%)$ & & 0 & 0 & \\
\hline
\end{tabular}

Abbreviation: NA not applicable, TRG tumor regression grade

The $p$ value in boldface means statistically significant, that is, less than 0.05

were associated with a high adequate rate of downstaging (Table 3). The patients with tumor length $\leq 4 \mathrm{~cm}$ $(P=0.044)$ or CEA exponential decrease $(P=0.008)$ potentially achieved a pCR (Table 4). Other clinical factors such as age, gender, pre-treatment $\mathrm{N}$ stage, distance from the anal verge, pre- or post-treatment 
Table 3 Univariate analysis of predictors for tumor downstaging in the training and validation cohorts

\begin{tabular}{|c|c|c|c|c|c|c|}
\hline \multirow[t]{2}{*}{ Variable } & \multicolumn{3}{|l|}{ Training cohort } & \multicolumn{3}{|l|}{ Validation cohort } \\
\hline & Good downstaging & Poor downstaging & $P$ & Good downstaging & Poor downstaging & $P$ \\
\hline No. of patients & 20 & 51 & & 26 & 49 & \\
\hline Age (mean, y $\pm S D$ ) & $58 \pm 10$ & $52 \pm 13$ & 0.101 & $54 \pm 10$ & $55 \pm 13$ & 0.630 \\
\hline Gender & & & 0.671 & & & 0.701 \\
\hline Male & $14(70 \%)$ & $33(65 \%)$ & & $11(42 \%)$ & $23(47 \%)$ & \\
\hline Female & $6(30 \%)$ & $18(35 \%)$ & & $15(58 \%)$ & $26(53 \%)$ & \\
\hline Pretreatment T stage & & & 0.009 & & & 0.125 \\
\hline $\mathrm{T}_{2}$ & 0 & 0 & & $2(8 \%)$ & $4(8 \%)$ & \\
\hline$T_{3}$ & 19 (95\%) & $33(65 \%)$ & & $24(92 \%)$ & $38(78 \%)$ & \\
\hline $\mathrm{T}_{4}$ & $1(5 \%)$ & $18(35)$ & & 0 & $7(14 \%)$ & \\
\hline Pretreatment $\mathrm{N}$ stage & & & 0.160 & & & 0.858 \\
\hline $\mathrm{N}_{0}$ & $2(10 \%)$ & $10(20 \%)$ & & $8(31 \%)$ & $13(26 \%)$ & \\
\hline$N_{1}$ & $12(60 \%)$ & $18(35 \%)$ & & $12(46 \%)$ & $22(45 \%)$ & \\
\hline $\mathrm{N}_{2}$ & $6(30 \%))$ & $23(45 \%)$ & & $6(23 \%)$ & $14(29 \%)$ & \\
\hline Tumor differentiation & & & 0.020 & & & 0.818 \\
\hline Well/Moderately & $14(70 \%)$ & $20(40 \%)$ & & 19 (73\%) & 37 (76\%) & \\
\hline Poorly/Undifferentiated & $6(30 \%)$ & $31(60 \%)$ & & $7(27 \%)$ & $12(24 \%)$ & \\
\hline Distance from the anal verge & & & 0.327 & & & 0.235 \\
\hline$\leqq 5 \mathrm{~cm}$ & $8(40 \%)$ & $27(53 \%)$ & & $8(31 \%)$ & $22(45 \%)$ & \\
\hline$>5 \mathrm{~cm}$ & $12(60 \%)$ & $24(47)$ & & $18(69 \%)$ & $27(55 \%)$ & \\
\hline Tumor length & & & 0.058 & & & 0.267 \\
\hline$\leqq 4 \mathrm{~cm}$ & $12(60 \%)$ & $18(65 \%)$ & & $13(50 \%)$ & $18(37 \%)$ & \\
\hline$>4 \mathrm{~cm}$ & $8(40 \%)$ & $33(35 \%)$ & & $13(50 \%)$ & $31(63 \%)$ & \\
\hline Circumferential extent & & & 0.065 & & & 0.856 \\
\hline$\leqq 50 \%$ & $8(40 \%)$ & $9(18 \%)$ & & $9(35 \%)$ & $18(37 \%)$ & \\
\hline$>50 \%$ & $12(60 \%)$ & $42(82 \%)$ & & $17(65 \%)$ & $31(63 \%)$ & \\
\hline Pre- treatment CEA level (mean, ng/mL; $95 \% \mathrm{Cl}$ ) & $23(14-33)$ & $28(19-39)$ & 0.551 & $17(12-22)$ & $13(11-15)$ & 0.066 \\
\hline Post- treatment CEA level (mean, $\mathrm{ng} / \mathrm{mL} ; 95 \% \mathrm{Cl}$ ) & $5(0.2-9)$ & $6(4-8)$ & 0.680 & $6(4-9)$ & $6(4-7)$ & 0.712 \\
\hline Normalization of post- treatment CEA Level & & & 0.124 & & & 0.600 \\
\hline Normal $(<5 \mathrm{ng} / \mathrm{mL})$ & $16(80 \%)$ & $31(61 \%)$ & & $17(65 \%)$ & $29(60 \%)$ & \\
\hline Elevated ( $\geqq 5 \mathrm{ng} / \mathrm{mL})$ & $4(20 \%)$ & $20(39 \%)$ & & $9(35 \%)$ & $20(40 \%)$ & \\
\hline CEA clearance pattern & & & 0.001 & & & 0.007 \\
\hline Exponential decrease $\left(R^{2} \geqq 0.9\right)$ & $15(75 \%)$ & $16(31 \%)$ & & $18(69 \%)$ & $18(37 \%)$ & \\
\hline Non-exponential decrease $\left(R^{2}<0.9\right)$ & $5(25 \%)$ & $35(69 \%)$ & & $8(31 \%)$ & $31(63 \%)$ & \\
\hline Concurrent chemotherapy & & & 0.601 & & & NA \\
\hline Capecitabine & 0 & 0 & & $26(35 \%)$ & $49(65 \%)$ & \\
\hline Infusional fluorouracil & $10(50 \%)$ & $29(57 \%)$ & & 0 & 0 & \\
\hline mFOLFOX6 & $10(50 \%)$ & $22(43 \%)$ & & 0 & 0 & \\
\hline
\end{tabular}

Abbreviation: NA not applicable

The $p$ value in boldface means statistically significant, that is, less than 0.05

CEA level, and concurrent chemotherapy regimen exerted no predictive significance for TRG, tumor downstaging, and pCR.

According to the multivariate analysis, the CEA clearance pattern was independent predictive factor for tumor downstaging [Odds ratio (OR), 8.25; 95\% CI 2.19-31.10; $P=0.002$; Table 5) and $\mathrm{pCR}(\mathrm{OR}, 8.30$; 95\% CI, 1.56-44.17; $P=0.013$ ) (Table 6). The AUC for the CEA clearance pattern was 0.63 (95\% CI, 0.50-0.76), 0.72 (95\% CI, 0.59-0.85), and 0.73 (95\% CI, 0.57-0.88), respectively (Fig. 2). 
Table 4 Univariate analysis of predictors for pCR in the training and validation cohorts

\begin{tabular}{|c|c|c|c|c|c|c|}
\hline \multirow[t]{2}{*}{ Variable } & \multicolumn{3}{|c|}{ Training cohort } & \multicolumn{3}{|c|}{ Validation cohort } \\
\hline & $\mathrm{pCR}$ & No $p C R$ & P & $\mathrm{pCR}$ & No $p C R$ & $P$ \\
\hline No. of patients & 11 & 60 & & 10 & 65 & \\
\hline Age (mean, y $\pm S D$ ) & $57 \pm 12$ & $54 \pm 13$ & 0.323 & $55 \pm 13$ & $55 \pm 12$ & 0.910 \\
\hline Gender & & & 0.618 & & & 0.497 \\
\hline Male & $8(73 \%)$ & $39(65 \%)$ & & $6(60 \%)$ & $28(43 \%)$ & \\
\hline Female & $3(27 \%)$ & $21(35 \%)$ & & $4(40 \%)$ & 37 (57\%) & \\
\hline Pretreatment T stage & & & 0.267 & & & 0.546 \\
\hline $\mathrm{T}_{2}$ & 0 & 0 & & $1(10 \%)$ & $5(8 \%)$ & \\
\hline $\mathrm{T}_{3}$ & $10(9 \%)$ & $42(70 \%)$ & & $9(90 \%)$ & $53(81 \%)$ & \\
\hline $\mathrm{T}_{4}$ & $1(91 \%)$ & $18(30 \%)$ & & 0 & $7(11 \%)$ & \\
\hline Pretreatment N stage & & & 0.217 & & & 0.121 \\
\hline $\mathrm{N}_{0}$ & $2(18 \%)$ & $10(17 \%)$ & & $4(40 \%)$ & $17(26 \%)$ & \\
\hline $\mathrm{N}_{1}$ & $7(64 \%)$ & $23(38 \%)$ & & $6(60 \%)$ & $28(43 \%)$ & \\
\hline $\mathrm{N}_{2}$ & $2(18 \%)$ & $27(45 \%)$ & & 0 & $20(31 \%)$ & \\
\hline Tumor differentiation & & & 0.073 & & & 0.707 \\
\hline Well/Moderately & $8(73 \%)$ & $26(43 \%)$ & & $7(70 \%)$ & 49 (75\%) & \\
\hline Poorly/Undifferentiated & $3(27 \%)$ & 34 (57\%) & & $3(30 \%)$ & $16(25 \%)$ & \\
\hline Distance from the anal verge & & & 0.301 & & & 0.731 \\
\hline$\leqq 5 \mathrm{~cm}$ & $7(64 \%)$ & $28(53 \%)$ & & $3(30 \%)$ & $27(42 \%)$ & \\
\hline$>5 \mathrm{~cm}$ & $4(36 \%)$ & $32(47 \%)$ & & $7(70 \%)$ & $38(58 \%)$ & \\
\hline Tumor length & & & 0.044 & & & 0.509 \\
\hline$\leqq 4 \mathrm{~cm}$ & $8(73 \%)$ & $22(37 \%)$ & & $3(30 \%)$ & $28(43 \%)$ & \\
\hline$>4 \mathrm{~cm}$ & $3(27 \%)$ & $38(63 \%)$ & & $7(70 \%)$ & $37(57 \%)$ & \\
\hline Circumferential extent & & & 0.441 & & & 0.480 \\
\hline$\leqq 50 \%$ & $4(36 \%)$ & $13(22 \%)$ & & $5(50 \%)$ & $22(34 \%)$ & \\
\hline$>50 \%$ & $7(64 \%)$ & $47(78 \%)$ & & $5(50 \%)$ & $43(66 \%)$ & \\
\hline Pre-treatment CEA level (mean, ng/mL; 95\%Cl) & $24(10-38)$ & $28(19-37)$ & 0.709 & $13(11-15)$ & $20(10-30)$ & 0.165 \\
\hline Post- treatment CEA level (mean, ng/mL; 95\%Cl) & $2(0.2-4)$ & $6(4-8)$ & 0.164 & $6(2-11)$ & $6(5-7)$ & 0.808 \\
\hline Normalization of post- treatment CEA Level & & & 0.312 & & & 1.000 \\
\hline Normal (<5 ng/mL) & $9(82 \%)$ & $38(63 \%)$ & & $6(60 \%)$ & $40(62 \%)$ & \\
\hline Elevated $(\geqq 5 \mathrm{ng} / \mathrm{mL})$ & $2(18 \%)$ & $22(37 \%)$ & & $4(40 \%)$ & $25(38 \%)$ & \\
\hline CEA clearance pattern & & & 0.008 & & & 0.042 \\
\hline Exponential decrease $\left(R^{2} \geqq 0.9\right)$ & $9(82 \%)$ & $22(37 \%)$ & & $8(80 \%)$ & $28(43 \%)$ & \\
\hline Non-exponential decrease $\left(R^{2}<0.9\right)$ & $2(18 \%)$ & $38(63 \%)$ & & $2(20 \%)$ & $37(57 \%)$ & \\
\hline Concurrent chemotherapy & & & 0.743 & & & NA \\
\hline Capecitabine & 0 & 0 & & $10(13 \%)$ & $65(87 \%)$ & \\
\hline Infusional fluorouracil & $7(64 \%)$ & $32(47 \%)$ & & 0 & 0 & \\
\hline mFOLFOX6 & $4(36 \%)$ & 28 (53\%) & & 0 & 0 & \\
\hline
\end{tabular}

Abbreviation: NA not applicable, $p C R$ pathologic complete response

The $p$ value in boldface means statistically significant, that is, less than 0.05

\section{Validation of CEA clearance pattern in an independent} cohort

Forty four patients (44\%) exhibited TRG 0-1, and 26 (35\%) had good downstaging. A pCR was observed in 10 patients (13\%). The CEA clearance pattern served as a predictor of tumor response to neoadjuvant treatment, which was further confirmed in an independent validation cohort of 75 patients. These results were similar to those obtained from the training cohort (Tables 2, 3, 4). The exponential decrease of CEA was associated with 
Table 5 Multivariate regression analysis with tumor downstaging as dependent variable

\begin{tabular}{|c|c|c|c|c|}
\hline Variable & Good downstaging & Poor downstaging & $P$ & Odds Ratio $(95 \% \mathrm{Cl})$ \\
\hline \multicolumn{5}{|l|}{ Training cohort } \\
\hline Pretreatment T stage & & & 0.04 & $10.14(1.11-92.55)$ \\
\hline $\mathrm{T} 2-3$ & $19(95 \%)$ & $33(65 \%)$ & & \\
\hline T4 & $1(5 \%)$ & $18(35)$ & & \\
\hline Tumor differentiation & & & 0.032 & $4.21(1.13-15.68)$ \\
\hline Well/Moderately & $14(70 \%)$ & $20(40 \%)$ & & \\
\hline Poorly/Undifferentiated & $6(30 \%)$ & $31(60 \%)$ & & \\
\hline CEA clearance pattern & & & 0.002 & $8.25(2.19-31.10)$ \\
\hline Exponential decrease $\left(R^{2} \geqq 0.9\right)$ & $15(75 \%)$ & $16(31 \%)$ & & \\
\hline Non-exponential decrease $\left(R^{2}<0.9\right)$ & $5(25 \%)$ & $35(69 \%)$ & & \\
\hline \multicolumn{5}{|l|}{ Validation cohort } \\
\hline Pretreatment T stage & & & 0.108 & $0.31(0.08-1.29)$ \\
\hline T2-3 & $26(78 \%)$ & $42(86 \%)$ & & \\
\hline T4 & 0 & $7(14 \%)$ & & \\
\hline Tumor differentiation & & & 0.752 & $1.22(0.36-4.09)$ \\
\hline Well/Moderately & 19 (73\%) & 37 (76\%) & & \\
\hline Poorly/Undifferentiated & $7(27 \%)$ & $12(24 \%)$ & & \\
\hline CEA clearance pattern & & & 0.008 & $4.28(1.47-12.43)$ \\
\hline Exponential decrease $\left(R^{2} \geqq 0.9\right)$ & $18(69 \%)$ & $18(37 \%)$ & & \\
\hline Non-exponential decrease $\left(R^{2}<0.9\right)$ & $8(31 \%)$ & $31(63 \%)$ & & \\
\hline
\end{tabular}

The $p$ value in boldface means statistically significant, that is, less than 0.05

TRG 0-1 $(P=0.016)$, good downstaging $(P=0.007)$, and pCR $(P=0.042)$.

In addition, the multivariate analysis considered the clinical parameters same as those in the training cohort. The CEA clearance pattern remained a significant predictive factor for TRG (OR, 4.37; 95\% CI,
1.44-13.23; $P=0.009 ;$ Table 7), tumor downstaging (OR, 4.28; 95\% CI, 1.47-12.43; $P=0.008$; Table 5), and $\mathrm{pCR}$ (OR, 5.22; 95\% CI, 1.02-26.60; $P=0.047$; Table 6). The ability of discrimination of the CEA clearance pattern, as assessed by AUC, was 0.64 (95\% $\mathrm{CI}, 0.51-0.77), 0.66$ (95\% CI, 0.53-0.79), and 0.69

Table 6 Multivariate regression analysis with $\mathrm{pCR}$ as dependent variable

\begin{tabular}{|c|c|c|c|c|}
\hline Variable & $\mathrm{pCR}$ & No $p C R$ & P & Odds Ratio $(95 \% \mathrm{Cl}$ \\
\hline \multicolumn{5}{|l|}{ Training cohort } \\
\hline Tumor length & & & 0.037 & $4.99(1.10-22.63)$ \\
\hline$\leqq 4 \mathrm{~cm}$ & $8(73 \%)$ & $22(37 \%)$ & & \\
\hline$>4 \mathrm{~cm}$ & $3(27 \%)$ & $38(63 \%)$ & & \\
\hline CEA clearance pattern & & & 0.013 & $8.30(1.56-44.17)$ \\
\hline Exponential decrease $\left(R^{2} \geqq 0.9\right)$ & $9(82 \%)$ & $22(37 \%)$ & & \\
\hline Non-exponential decrease $\left(R^{2}<0.9\right)$ & $2(18 \%)$ & $38(63 \%)$ & & \\
\hline \multicolumn{5}{|l|}{ Validation cohort } \\
\hline Tumor length & & & 0.484 & $0.59(0.13-2.59)$ \\
\hline$\leqq 4 \mathrm{~cm}$ & $3(30 \%)$ & $28(43 \%)$ & & \\
\hline$>4 \mathrm{~cm}$ & $7(70 \%)$ & $37(57 \%)$ & & \\
\hline CEA clearance pattern & & & 0.047 & $5.22(1.02-26.60)$ \\
\hline Exponential decrease $\left(R^{2} \geqq 0.9\right)$ & $8(80 \%)$ & $28(43 \%)$ & & \\
\hline Non-exponential decrease $\left(R^{2}<0.9\right)$ & $2(20 \%)$ & 37 (57\%) & & \\
\hline
\end{tabular}



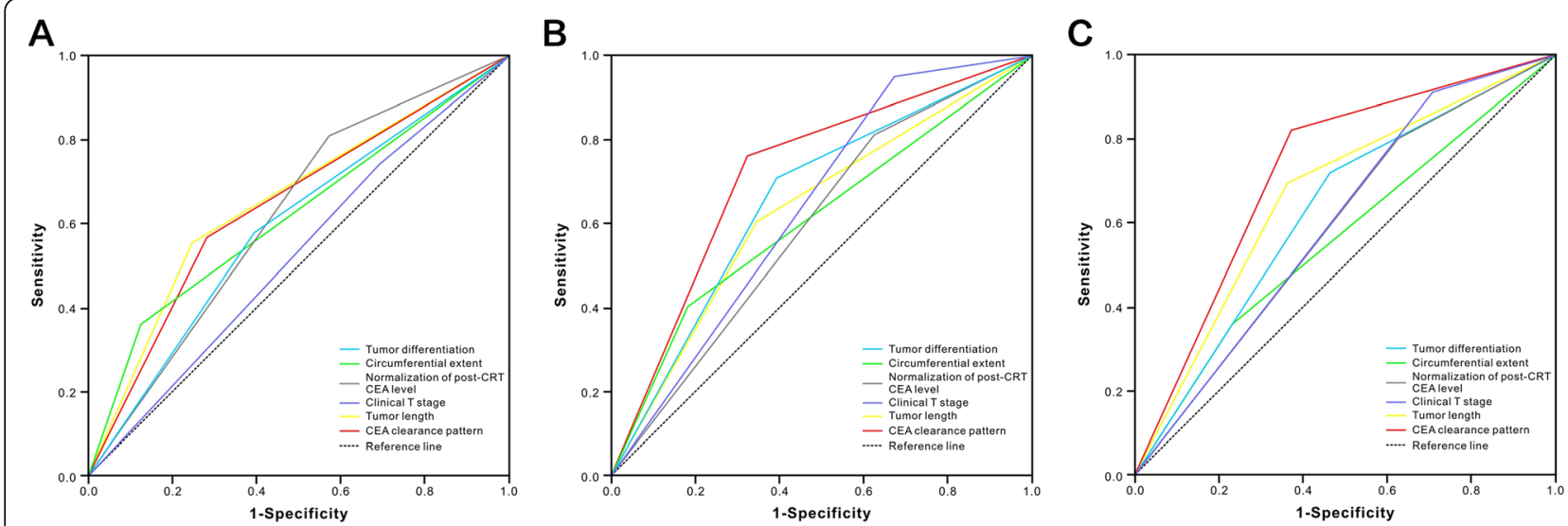

Fig. 2 Predictive ability of the CEA clearance pattern for TRG (a), tumor downstaging (b), and pCR (c) were compared to the other clinical parameters by AUC curves in the training cohort

Table 7 Multivariate regression analysis with TRG as dependent variable

\begin{tabular}{|c|c|c|c|c|}
\hline Variable & TRG 0-1 & TRG 2-3 & $P$ & Odds Ratio $(95 \% \mathrm{Cl})$ \\
\hline \multicolumn{5}{|l|}{ Training cohort } \\
\hline Tumor length & & & 0.037 & $3.19(1.07-9.46)$ \\
\hline$\leqq 4 \mathrm{~cm}$ & $19(42 \%)$ & $11(71 \%)$ & & \\
\hline$>4 \mathrm{~cm}$ & $14(58 \%)$ & $27(29 \%)$ & & \\
\hline Circumferential extent & & & 0.146 & $2.65(0.71-9.86)$ \\
\hline$\leqq 50 \%$ & $12(36 \%)$ & $5(13 \%)$ & & \\
\hline$>50 \%$ & $21(64 \%)$ & $33(87 \%)$ & & \\
\hline Normalization of post- treatment CEA Level & & & 0.082 & $2.81(0.88-9.01)$ \\
\hline Normal (<5 ng/mL) & $26(79 \%)$ & $21(55 \%)$ & & \\
\hline Elevated $(\geqq 5 \mathrm{ng} / \mathrm{mL})$ & $7(21 \%)$ & $17(45 \%)$ & & \\
\hline CEA clearance pattern & & & 0.161 & $2.18(0.73-6.44)$ \\
\hline Exponential decrease $\left(R^{2} \geqq 0.9\right)$ & $19(58 \%)$ & $12(32 \%)$ & & \\
\hline Non-exponential decrease $\left(R^{2}<0.9\right)$ & $14(42 \%)$ & $26(68 \%)$ & & \\
\hline \multicolumn{5}{|l|}{ Validation cohort } \\
\hline Tumor length & & & 0.009 & $4.23(1.43-12.50)$ \\
\hline$\leqq 4 \mathrm{~cm}$ & $19(58 \%)$ & $12(29 \%)$ & & \\
\hline$>4 \mathrm{~cm}$ & $14(42 \%)$ & $30(64 \%)$ & & \\
\hline Circumferential extent & & & 0.147 & $0.42(0.13-1.35)$ \\
\hline$\leqq 50 \%$ & $9(27 \%)$ & $18(43 \%)$ & & \\
\hline$>50 \%$ & $24(73 \%)$ & $24(57 \%)$ & & \\
\hline Normalization of post- treatment CEA Level & & & 0.884 & $0.92(0.29-2.92)$ \\
\hline Normal (<5 ng/mL) & $23(70 \%)$ & $23(55 \%)$ & & \\
\hline Elevated ( $\geqq 5 \mathrm{ng} / \mathrm{mL}$ ) & $10(30 \%)$ & $19(45 \%)$ & & \\
\hline CEA clearance pattern & & & 0.009 & $4.37(1.44-13.23)$ \\
\hline Exponential decrease $\left(R^{2} \geqq 0.9\right)$ & $21(64 \%)$ & $15(36 \%)$ & & \\
\hline Non-exponential decrease $\left(R^{2}<0.9\right)$ & $12(36 \%)$ & $27(64 \%)$ & & \\
\hline
\end{tabular}


(95\% CI, 0.52-0.85) for TRG, tumor downstaging, and pCR (Fig. 3), respectively.

\section{Discussion}

CEA is the most widely used and readily available tumor marker for the management of colorectal cancer. The diagnostic and prognostic value of this marker has been extensively evaluated [23-27]. Recent studies have investigated its potential predictive value in tumor response to neoadjuvant treatment of rectal cancer. Wallin et al. recruited 530 patients and found that low pre-treatment CEA (3.4 vs. $9.6 \mathrm{ng} / \mathrm{mL}$ ) was significantly associated with $\mathrm{pCR}$ in the multivariate analysis. When stratifying for smoking status, the predictive value was significant only in the nonsmokers [28]. Huh et al. demonstrated that low pre-treatment CEA level $(<$ $5 \mathrm{ng} / \mathrm{mL}$ ), non-circumferentiality, and non-macroscopic ulceration comprised of the independent predictors of a high pCR rate [9]. Yang et al. showed that low post-treatment CEA levels could predict of pCR. Especially, using ROC curves, the study determined that $2.61 \mathrm{ng} / \mathrm{mL}$ was the optimal cut-off level for CEA with a sensitivity and specificity of 76 and $58.4 \%$, respectively [16]. Perez et al. demonstrated that post-treatment CEA levels $<5 \mathrm{ng} / \mathrm{mL}$ were predictive of both pCR as well as 5-year overall survival [14]. Kleiman et al. demonstrated that the normalization of post-treatment CEA in patients with elevated pretreatment CEA levels was a significant predictor of pCR resulting in an approximately 65 -fold potential increase in achieving pCR [15].

Contrary to previous studies, we did not find that patients achieving an good TRG, satisfactory tumor downstaging, and $\mathrm{pCR}$ were significantly associated with preor post-treatment CEA levels and normalization of post-treatment CEA according to the multivariate analysis. Thus, we hypothesized that the pre-treatment CEA value only reflected the tumor burden up to a specific extent but not the efficacy of the neoadjuvant treatment. The post-treatment CEA values would be less capable of providing the tumor response to neoadjuvant treatment as compared to the dynamic changes in CEA levels as they represent only one time point. Moreover, Kim et al. demonstrated that the pattern of serum CEA clearance after radical tumor resection in rectal cancer patients could be used as a surrogate marker for predicting cancer-specific mortality [22].

To the best of our knowledge, this is the first study evaluating the dynamic changes in CEA levels during neoadjuvant treatment that serves as a predictor for rectal cancer patients in two independent cohorts. In the present study, the CEA clearance pattern was constructed and categorized based on the $\mathrm{R}^{2}$ value, which was calculated by drawing the exponential trend lines using serially estimated CEA; this method was similar to that of Kim et al. [22]. Furthermore, our data showed that the patients in the CEA exponential decrease group defined by $R^{2}>0.9$ had significantly good TRG, good tumor downstaging, and pCR rates after neoadjuvant treatment as assessed in a cohort of patients in the FOWARC trial [17]. A similar predictive effect of CEA clearance pattern on tumor response to neoadjuvant treatment was validated in an independent consecutive retrospective cohort. The predictive ability of the both cohort was stronger than that of the pre- or post-treatment CEA levels, normalization of CEA levels, and other conventional variables such as pre-treatment $\mathrm{T}$ or $\mathrm{N}$ stage, tumor differentiation, distance from the anal verge, tumor length, and circumferential extent.

According to the response evaluation criteria in solid tumors (RECIST version 1.1), if markers are initially above the upper normal limit, one of the criteria for assessing clinical complete response for a patient is the reduction of tumor markers to normal levels. Our study
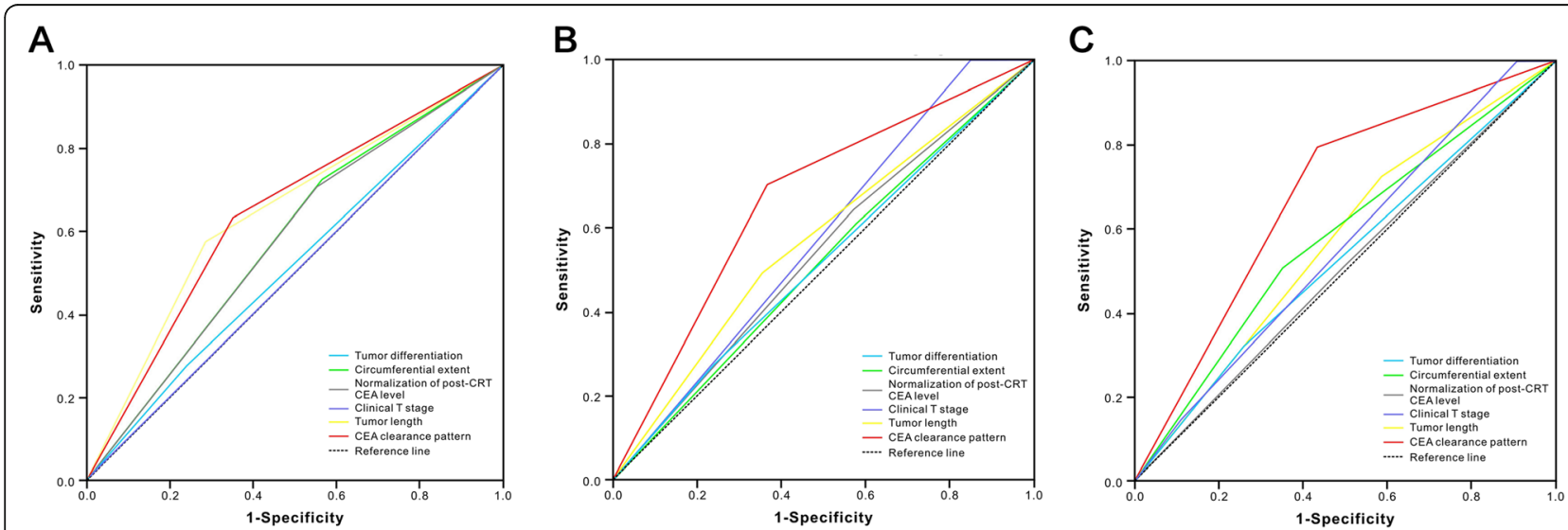

Fig. 3 Predictive ability of the CEA clearance pattern for TRG (a), tumor downstaging (b), and pCR (c) were compared to the other clinical parameters by AUC curves in the validation cohort 
demonstrated that the pattern of CEA clearance might substitute for normalization of CEA level and be used as an adjunct in the assessment of complete clinical response that can guide individualized treatment strategies such as "Watch \& Wait" [29-31]. In addition, the measure of CEA clearance pattern was established according to the baseline and the follow-up CEA values during neoadjuvant treatment, which are normally available in the clinical practice.

Nevertheless, the limitations of our study are as follows: 1) In the both training and validation cohort, baseline and follow-up CEA data could be evaluated only for a partial study population and in a single institution; 2) In the training cohort, the exponential decrease of CEA was significantly associated with good TRG as assessed by univariate analysis; however, statistical significance was not observed by multivariate analysis. These inconsistent results could be attributed to small sample sizes and the quality of retrospective cohort; 3) CEA is known to be elevated in smokers, and therefore a different reference value for a positive test is used. When assessing the predictive value of CEA dynamic changes, the influence of the patient's smoking status is unclear. There is a potential negative effect of smoking on the efficacy of neoadjuvant chemoradiotherapy, however, we did not take into account the possible interaction of CEA clearance pattern with smoking due to absence of data.

\section{Conclusion}

Our results demonstrated the CEA clearance pattern, a low cost and reproducible clinical parameter, was an independent predictor of tumor response to neoadjuvant treatment in patients with rectal cancer. It might serve as an adjunct in the assessment of complete clinical response and guide individualized treatment strategies.

\section{Additional files}

Additional file 1: Figure S1. Schematic representation of the eligible patients included in the training cohort. (JPG $133 \mathrm{~kb}$ )

Additional file 2: Figure S2. Schematic representation of the eligible patients included in the validation cohort. (JPG $137 \mathrm{~kb}$ )

Additional file 3: Table S1. Correlation between the CEA clearance pattern and clinicopathological parameters. (DOCX $22 \mathrm{~kb}$ )

\section{Abbreviations \\ AUC: The area under the curves; CEA: Carcinoembryonic antigen; OR: Odds ratio; pCR: Pathologic complete response; RECIST: Response evaluation criteria in solid tumors; TME: Total mesorectal excision;" TRG: Tumor regression grade}

\section{Acknowledgments}

The authors thank Jianwei Zhang and Xiuping Deng for supplying patients, maintaining the database and obtaining follow-up data.

\section{Funding}

Not applicable.

\section{Availability of data and materials}

All data generated or analysed during this study are included in this published article and its supplementary information files.

\section{Authors' contributions}

All authors read and approved the final manuscript. $\mathrm{HBH}$ and JH: contributed to the data analysis and interpretation, drafting and revising the manuscript. $\mathrm{PL}, \mathrm{LW}$ and MJH: contributed to the data acquisition, critical revision for intellectual content. JPW and YHD: contributed to the study conception design, critical revision for intellectual content.

\section{Ethics approval and consent to participate}

The institutional review board (IRB) of The Sixth Affiliated Hospital of Sun Yat-Sen University approved the present study (Approval number: 06-08-2010) and all documented consent from all patients who participated in the study.

\section{Consent for publication}

Not applicable.

\section{Competing interests}

The authors declare that they have no competing interests.

\section{Publisher's Note}

Springer Nature remains neutral with regard to jurisdictional claims in published maps and institutional affiliations.

\section{Author details}

'Department of Medical Oncology, The Sixth Affiliated Hospital of Sun Yat-Sen University, Yuancunheng 2nd Road, Guangzhou 510655, People's Republic of China. ${ }^{2}$ Department of Oncology, Xiangya Hospital of Central South University, Changsha, China. ${ }^{3}$ Department of Colorectal Surgery, The Sixth Affiliated Hospital of Sun Yat-Sen University, Yuancunheng 2nd Road, Guangzhou 510655, People's Republic of China. ${ }^{4}$ Guangdong Institute of Gastroenterology, Guangdong Provincial Key Laboratory of Colorectal and Pelvic Floor Diseases, Guangzhou, China.

Received: 6 September 2018 Accepted: 25 October 2018

Published online: 20 November 2018

\section{References}

1. Arbman G, Nilsson E, Hallbook O, Sjodahl R. Local recurrence following total mesorectal excision for rectal cancer. Br J Surg. 1996;83(3):375-9.

2. Heald RJ, Ryall RD. Recurrence and survival after total mesorectal excision for rectal cancer. LANCET. 1986;1(8496):1479-82.

3. Roh MS, Colangelo LH, O'Connell MJ, Yothers G, Deutsch M, Allegra CJ, Kahlenberg MS, Baez-Diaz L, Ursiny CS, Petrelli NJ, et al. Preoperative multimodality therapy improves disease-free survival in patients with carcinoma of the rectum: NSABP R-03. J Clin Oncol. 2009;27(31):5124-30.

4. Sauer R, Becker H, Hohenberger W, Rodel C, Wittekind C, Fietkau R, Martus P, Tschmelitsch J, Hager E, Hess CF, et al. Preoperative versus postoperative chemoradiotherapy for rectal cancer. N Engl J Med. 2004;351(17):1731-40.

5. Park IJ, You YN, Agarwal A, Skibber JM, Rodriguez-Bigas MA, Eng C, Feig BW Das $\mathrm{P}$, Krishnan $\mathrm{S}$, Crane $\mathrm{CH}$, et al. Neoadjuvant treatment response as an early response indicator for patients with rectal cancer. J Clin Oncol. 2012; 30(15):1770-6

6. Martin ST, Heneghan HM, Winter DC. Systematic review and meta-analysis of outcomes following pathological complete response to neoadjuvant chemoradiotherapy for rectal cancer. Br J Surg. 2012;99(7):918-28.

7. Das P, Skibber JM, Rodriguez-Bigas MA, Feig BW, Chang GJ, Wolff RA, Eng C, Krishnan S, Janjan NA, Crane $\mathrm{CH}$. Predictors of tumor response and downstaging in patients who receive preoperative chemoradiation for rectal cancer. CANCER-AM CANCER SOC. 2007;109(9):1750-5.

8. van Stiphout RG, Lammering G, Buijsen J, Janssen MH, Gambacorta MA Slagmolen P, Lambrecht M, Rubello D, Gava M, Giordano A, et al. Development and external validation of a predictive model for pathological complete response of rectal cancer patients including sequential PET-CT imaging. Radiother Oncol. 2011;98(1):126-33.

9. Huh JW, Kim HR, Kim YJ. Clinical prediction of pathological complete response after preoperative chemoradiotherapy for rectal cancer. Dis Colon Rectum. 2013;56(6):698-703. 
10. Zhang J, Cai Y, Hu H, Lan P, Wang L, Huang M, Kang L, Wu X, Wang H, Ling $J$, et al. Nomogram basing pre-treatment parameters predicting early response for locally advanced rectal cancer with neoadjuvant chemotherapy alone: a subgroup efficacy analysis of FOWARC study. ONCOTARGET. 2016;7(4):5053-62.

11. Jayanand SB, Seshadri RA, Tapkire R. Signet ring cell histology and noncircumferential tumors predict pathological complete response following neoadjuvant chemoradiation in rectal cancers. Int J Color Dis. 2011;26(1):23-7

12. Kalady MF, de Campos-Lobato LF, Stocchi L, Geisler DP, Dietz D, Lavery IC, Fazio WW. Predictive factors of pathologic complete response after neoadjuvant chemoradiation for rectal cancer. Ann Surg. 2009;250(4):582-9.

13. GOLD P. Freedman so: demonstration of tumor-specific antigens in human colonic CARCINOMATA by immunological tolerance and absorption techniques. J Exp Med. 1965;121:439-62.

14. Perez RO, Sao JG, Habr-Gama A, Kiss D, Proscurshim I, Campos FG, GamaRodrigues JJ, Cecconello I. The role of carcinoembriogenic antigen in predicting response and survival to neoadjuvant chemoradiotherapy for distal rectal cancer. Dis Colon Rectum. 2009;52(6):1137-43.

15. Kleiman A, Al-Khamis A, Farsi A, Kezouh A, Vuong T, Gordon PH, Vasilevsky CA, Morin N, Faria J, Ghitulescu G, et al. Normalization of CEA levels postneoadjuvant therapy is a strong predictor of pathologic complete response in rectal Cancer. J Gastrointest Surg. 2015;19(6):1106-12.

16. Yang KL, Yang SH, Liang WY, Kuo YJ, Lin JK, Lin TC, Chen WS, Jiang JK, Wang HS, Chang SC, et al. Carcinoembryonic antigen (CEA) level, CEA ratio, and treatment outcome of rectal cancer patients receiving pre-operative chemoradiation and surgery. Radiat Oncol. 2013;8:43.

17. Deng Y, Chi P, Lan P, Wang L, Chen W, Cui L, Chen D, Cao J, Wei H, Peng X, et al. Modified FOLFOX6 with or without radiation versus fluorouracil and Leucovorin with radiation in neoadjuvant treatment of locally advanced rectal Cancer: initial results of the Chinese FOWARC multicenter, open-label, randomized three-arm phase III trial. J Clin Oncol. 2016;34(27):3300-7.

18. Ryan R, Gibbons D, Hyland JM, Treanor D, White A, Mulcahy HE, O'Donoghue DP, Moriarty M, Fennelly D, Sheahan K. Pathological response following long-course neoadjuvant chemoradiotherapy for locally advanced rectal cancer. HISTOPATHOLOGY. 2005;47(2):141-6.

19. D'Amico AV, Moul JW, Carroll PR, Sun L, Lubeck D, Chen MH. Surrogate end point for prostate cancer-specific mortality after radical prostatectomy or radiation therapy. J Natl Cancer Inst. 2003;95(18):1376-83.

20. Spiess PE, Lee AK, Leibovici D, Wang X, Do KA, Pisters LL. Presalvage prostate-specific antigen (PSA) and PSA doubling time as predictors of biochemical failure of salvage cryotherapy in patients with locally recurrent prostate cancer after radiotherapy. CANCER-AM CANCER SOC. 2006;107(2): 275-80.

21. Yoshimasu T, Maebeya S, Suzuma T, Bessho T, Tanino H, Arimoto J, Sakurai T, Naito Y. Disappearance curves for tumor markers after resection of intrathoracic malignancies. Int J Biol Markers. 1999;14(2):99-105.

22. Kim JY, Kim NK, Sohn SK, Kim YW, Kim KJ, Hur H, Min BS, Cho CH. Prognostic value of postoperative CEA clearance in rectal cancer patients with high preoperative CEA levels. Ann Surg Oncol. 2009;16(10):2771-8.

23. Choi JS, Min JS. Significance of postoperative serum level of carcinoembryonic antigen (CEA) and actual half life of CEA in colorectal cancer patients. Yonsei Med J. 1997;38(1):1-7.

24. Arnaud JP, Koehl C, Adloff M. Carcinoembryonic antigen (CEA) in diagnosis and prognosis of colorectal carcinoma. Dis Colon Rectum. 1980;23(3):141-4.

25. Harrison LE, Guillem JG, Paty P, Cohen AM. Preoperative carcinoembryonic antigen predicts outcomes in node-negative colon cancer patients: a multivariate analysis of 572 patients. J Am Coll Surg. 1997;185(1):55-9.

26. Park YA, Lee KY, Kim NK, Baik SH, Sohn SK, Cho CW. Prognostic effect of perioperative change of serum carcinoembryonic antigen level: a useful tool for detection of systemic recurrence in rectal cancer. Ann Surg Oncol. 2006;13(5):645-50

27. Wolmark N, Fisher B, Wieand HS, Henry RS, Lerner H, Legault-Poisson S, Deckers PJ, Dimitrov N, Gordon PH, Jochimsen P, et al. The prognostic significance of preoperative carcinoembryonic antigen levels in colorectal cancer. Results from NSABP (National Surgical Adjuvant Breast and bowel project) clinical trials. Ann Surg. 1984;199(4):375-82.

28. Wallin U, Rothenberger $D$, Lowry A, Luepker $R$, Mellgren A. CEA - a predictor for pathologic complete response after neoadjuvant therapy for rectal cancer. Dis Colon Rectum. 2013;56(7):859-68.
29. Maas M, Beets-Tan RG, Lambregts DM, Lammering G, Nelemans PJ, Engelen SM, van Dam RM, Jansen RL, Sosef M, Leijtens JW, et al. Wait-and-see policy for clinical complete responders after chemoradiation for rectal cancer. J Clin Oncol. 2011:29(35):4633-40.

30. Habr-Gama A, Perez RO, Nadalin W, Sabbaga J, Ribeiro UJ, Silva ESAJ, Campos FG, Kiss DR, Gama-Rodrigues J. Operative versus nonoperative treatment for stage 0 distal rectal cancer following chemoradiation therapy: long-term results. Ann Surg. 2004;240(4):711-7 717-718.

31. Probst CP, Becerra AZ, Aquina CT, Tejani MA, Hensley BJ, Gonzalez MG, Noyes K, Monson JR, Fleming FJ. Watch and wait?--elevated pretreatment CEA is associated with decreased pathological complete response in rectal Cancer. J Gastrointest Surg. 2016;20(1):43-52, 52.

\section{Ready to submit your research? Choose BMC and benefit from:}

- fast, convenient online submission

- thorough peer review by experienced researchers in your field

- rapid publication on acceptance

- support for research data, including large and complex data types

- gold Open Access which fosters wider collaboration and increased citations

- maximum visibility for your research: over $100 \mathrm{M}$ website views per year

At $\mathrm{BMC}$, research is always in progress.

Learn more biomedcentral.com/submissions 